\title{
Weak Domination in LICT Graphs
}

\author{
M. H. Muddebihal \\ Professor \\ Department of Mathematics, \\ Gulbarga University, \\ Kalaburagi-585106, \\ Karnataka, India
}

\author{
Geetadevi Baburao \\ Research Scholar \\ Department of Mathematics, \\ Gulbarga University, \\ Kalaburagi-585106, \\ Karnataka, India
}

\begin{abstract}
The lict graph $n(G)$ of a graph $G$ is the graph whose set of vertices is the union of set of edges and the set of cut vertices of $G$ in which two vertices are adjacent if and only if the corresponding edges are adjacent or the corresponding members of $G$ are incident.In this paper, we initiate the study of variation of standard domination, namely weak lict domination. A weak dominating set $\mathrm{D}$ is a weak dominating set of $n(G)$, if for every vertex $y \in V[n(G)]-D$ there is a vertex $\mathrm{x} \in \mathrm{D}$ with $\operatorname{deg}(\mathrm{x}) \leq \operatorname{deg}(\mathrm{y})$ and $\mathrm{y}$ is adjacent to $\mathrm{x}$. A weak domination number of $n(G)$ is denoted by $\gamma_{w n}(G)$, is the smallest cardinality of a weak dominating set of $n(G)$. We determine best possible upper and lower bounds for $\gamma_{w n}(G)$, in terms of elements of $G$.
\end{abstract}

\section{Keywords}

Domination, Double domination, restrained domination, weak domination, weak lict domination.

\section{Subject classification number: AMS-05C69, $05 \mathrm{C} 70$.}

\section{INTRODUCTION}

In this note, we will use the notation and terminology in [1].Let $G=(V, E)$ be a simple graph with $|V|=p$ and $|E|=q$. The degree, neighborhood and closed neighborhood of a vertex $v$ in a graph $G$ are denoted as $\operatorname{deg}(v), N(v)$ and $N[v]=N(v) \cup v$ respectively. We use $\Delta(G)\left[\Delta^{\prime}(G)\right]$ denotes the maximum degree of a vertex (edge)in $G$ and $\delta(G) \delta^{\prime}(G)$ denotes the minimum degree of a vertex (edge) in $G$. The notation $\alpha_{0}(G)\left[\alpha_{1}(G)\right]$ is the minimum number of vertices (edges) in a vertex (edge) covers of $G$. The notation $\beta_{0}(G)\left[\left(\beta_{1}\right)\right]$ is the minimum number of vertices (edges) in a maximal independent set of a vertex (edge) of $G$.

We say a set $S$ is a dominating set of $G$, if for every vertex $u \in V-S$, there exists a vertex $v \in S$ such that $u v \in E$. The domination number of $G$, denoted by $\gamma(G)$ is the minimum cardinality of a dominating set of $G$. For compressive work on the subject has been done in [3],[4].A dominating set $S$ of $G$ is called a connected dominating set of $G$, if the induced subgraph $\langle S\rangle$ is connected. The minimum cardinality taken over all the minimal connected domination number and is denoted by $\gamma_{c}(G)$. A dominating set $S \subseteq V(G)$ is called the total dominating set, if for every vertex $v \in V$, there exist a vertex $u \in S, u \neq v$ such that $u$ is adjacent to $v$. The total domination number of $G$ is denoted by $\gamma_{t}(G)$ is the minimum cardinality of total dominating set of $G$.A set $S$ of vertices in a graph $G$ is an independent dominating set of $G$, if $S$ is an independent set and every vertex not in $S$ is adjacent to a vertex in $S$. The smallest cardinality of an independent dominating set is called an independent domination number of $G$ and is denoted by $i(G)$.A dominating set $D$ of a graph $G$ is a split dominating set if the induced subgraph $\langle V-D\rangle$ is disconnected. The split domination number $\gamma_{S}(G)$ is the least cardinality of a split dominating set of $G$.

In [5], the strong slit domination was given as follows. A set $S \subseteq G$ is called the strong slit dominating set of $G$, if $\langle V-S\rangle$ is totally disconnected with at least two isolated vertices. The strong slit domination number $\gamma_{s S}(\mathrm{G})$ is the lowest level cardinality of minimal strong split dominating set of $G$.

A dominating set $D$ of a graph $G=(V, E)$ is a cotatal dominating set if, every vertex $v \in V-D$ is not an isolated vertex in the induced subgraph $\langle V-D\rangle$. The cototal domination number $\gamma_{\cot }(G)$ of $G$ is the smallest cardinality of a cototal dominating set of $G$.A set $S \subseteq V(G)$ is a Restrained dominating set of $G$, if every vertex in $V-S$ is adjacent to a vertex in $S$ as well as another vertex in $V-S$.The Restrained domination number of a graph $G$ is denoted by $\gamma_{\text {Res }}(G)$ is the least cardinality of a Restrained dominating set of $G$.Given two adjacent vertices $u$ and $v$ of $G$. We say $v$ weakly dominates $u$, if $\operatorname{deg}(v) \leq \operatorname{deg}(u)$. A set $S \subseteq V(G)$ is a weakly dominating set, if every vertex in $\langle V-S\rangle$ is weakly dominated by at least one vertex in $S$. Weak domination number $\gamma_{w}(G)$ is the minimum cardinality of a weak dominating set of $G$.

In [7], the author showed that a weak dominating set $S$ is a weak dominating set of $L(G)$, if for every vertex $u \in$ $V[L(G)]-S$ there is a vertex $v \in S$ with $\operatorname{deg}(v) \leq \operatorname{deg}(u)$ and $u$ is adjacent to $v$. A weak line domination number $\gamma_{w l}(G)$ is the least cardinality of a weak line dominating set $S$ of $L(G)$.

In [6], the author has defined that the lict graph $n(G)$ of a graph $G$ is the graph whose set of vertices is the union of set of edges and the set of cutvertices of $G$ in which two vertices are adjacent if and only if the corresponding edges are adjacent or corresponding members of $G$ are incident

In [8], Sampathkumar and Pushpa Latha have shown weak and strong domination number.

In this paper, we study the theoretic properties of $\gamma_{w n}(G)$ and many bounds are obtained in terms of elements of $G$ and its relationship with other domination parameter were found.

In section 2 we determine this parameter for some standard graphs. We obtained best possible upper and lower bound for $\gamma_{w n}(G)$

\section{RESULTS}

First we list out the exact values of $\gamma_{w n}(G)$ for some standard graphs.

Theorem 2.1: i].For any path $P_{n}$ with $n \geq 3$ vertices 
$\gamma_{w n}(G)=P-2$

ii].For any $\operatorname{star} K_{1, n}$ with $n \geq 2$ vertices

$$
\gamma_{w n}\left[K_{1, n}\right]=\gamma(G)=\gamma_{n}(G)=\gamma_{s}(G)=\gamma_{w}(G)=\gamma_{w l}=1 .
$$

iii]. For any cycle $C_{p}$ with $\mathrm{p} \geq 3$ vertices.

$$
\gamma_{w n}\left(C_{p}\right)=\left\{\begin{array}{c}
\frac{p}{3} \text { if } p \equiv O(\bmod 3) \\
\left\lceil\frac{p}{3}\right\rceil \text { otherwise } .
\end{array}\right.
$$

iv]. For any wheel $W_{p}$ with $p \geq 4$ vertices.

$$
\gamma_{w n}\left(W_{p}\right)=\left\{\begin{array}{l}
\frac{p}{2} \text { if } p \text { is even } \\
\left\lfloor\frac{p}{2}\right\rfloor \text { if } p \text { is odd }
\end{array}\right.
$$

v]. For any complete graph $K_{p}$ with $p \geq 3$ vertices.

$$
\gamma_{w n}\left(K_{p}\right)=\left\{\begin{array}{c}
\frac{p}{3} \text { if } p \equiv 0(\bmod 3) \\
\left\lceil\frac{p}{3}\right\rceil \text { Otherwise }
\end{array}\right.
$$

vi]. For any $\operatorname{star} K_{1, p}$ with $p \geq 2$ vertics $\gamma_{w n}\left(K_{1, p}\right)=\alpha_{0}$.

\section{Upper bounds for $\gamma_{w n}(G)$.}

We obtained an upper bound for $\gamma_{w n}(G)$ in terms of $\beta_{1}(G)$ and weak line domination $\gamma_{w l}(G)$.

Theorem 2.2: For any graph $G, \gamma_{w n}(G) \leq \gamma_{w l}(G)+\beta_{1}+$ 1.

Proof: Let $B=\left\{e_{1}, e_{2}, e_{3}, \ldots, e_{m}\right\} \subseteq E(G)$ with $N\left(e_{i}\right) \cap$ $N\left(e_{j}\right)=e$ for every $e_{i}, e_{j} \in B, \quad 1 \leq i \leq m, 1 \leq j \leq m$ and $e \in E(G)-B$. Clearly $B$ forms a maximal independent edge set in $G$.Let $A=\left\{u_{1}, u_{2}, u_{3}, \ldots, u_{n}\right\} \subseteq V[L(G)]$ be the set of vertices with $\operatorname{deg}\left(u_{i}\right) \geq 1,1 \leq i \leq n$. Such that $N[A]=$ $V[L(G)]$. Hence $A$ forms a $\gamma-\operatorname{set}$ of $L(G)$.

Suppose there exists a set $A_{1} \subseteq V[L(G)]-A$ such that $\forall u_{i} \in A_{1}, \operatorname{deg}\left(u_{i}\right) \geq \operatorname{deg}\left(u_{k}\right), \forall u_{k} \in A$. Then $A \cup A_{1}$ forms a minimal weak dominating set of $L(G)$.Suppose $S=\left\{u_{1} u_{2} u_{3}, \ldots, u_{n}\right\} \subseteq V[n(G)]$ and $\operatorname{deg}\left(u_{m}\right) \geq \operatorname{deg}\left(u_{n}\right)$, $\forall u_{m} \in V[n(G)]-S$ and $\forall u_{n} \in S$ such that $N[S]=V[n(G)]$. Then $S$ forms a dominating set of $n(G)$.Suppose there exists a set $S_{1} \subseteq V[n(G)]-S$ such that $u_{i} \in S \operatorname{deg}\left(u_{i}\right) \geq \operatorname{deg}\left(u_{k}\right)$, $\forall u_{k} \in S_{1}$. Then $S \cup S_{1}$ forms a minimal weak dominating set of $n(G)$. Hence it gives $\left|S \cup S_{1}\right| \leq\left|A \cup A_{1}\right|+|B|+1$. Clearly $\gamma_{w n}(G) \leq \gamma_{w l}(G)+\beta_{1}+1$.

A Roman domination function of a graph $G=(V, E)$ is a fuction $f: V \rightarrow\{0,1,2\}$ satisfying the condition that every vertex $u$ for which $f(u)=0$ is adjacent to at least one vertex $v$ for which $f(v)=2$. The weight of a Roman dominating function in $G$ is the value of $f(v)=\sum_{u \in v} f(u)$. The Roman domination number of $G$ is denoted by $\gamma_{R}(G)$, equals the smallest weight of a Roman dominating function on $G$. Farther we relates our concept to $\gamma_{R}(G)$ and $\alpha_{1}(G)$.

Theorem 2.3: For any connected $(p, q)$ graph $G$, with $(p \geq$ $2)$, then $\gamma_{w n}(G) \leq \alpha_{1}(G)+\gamma_{R}(G)+1$.

Proof: Let $f: V_{i} \rightarrow\{0,1,2\} ; i=0,1,2$ be a Roman domination function of $D^{\prime}$ where $D^{\prime}$ is a Roman dominating set of $G$. Let $A=\left\{e_{j}\right\}, j=1,2, \ldots, m$ be the minimal edge caver of $G$, so that $|A|=\alpha_{1}(G)$. Now in $n(G)$, let $B=$ $\left\{u_{1}, u_{2}, u_{3}, \ldots, u_{n}\right\} \subseteq V[n(G)]$ be the minimal set of vertices where $N[B]=V[n(G)]$. Then $B$ is a dominating set of $n(G)$. Suppose $\forall v_{i} \in V[n(G)]-B, \quad \operatorname{deg}\left(v_{i}\right) \leq$ $\operatorname{deg}\left(v_{k}\right)$ where $\forall v_{k} \in B$. Then $B \cup\left\{v_{i}\right\}$ forms a minimal weak lict $\gamma$-set of $G$. Thus $\left|B \cup\left\{v_{i}\right\}\right| \leq|A|+\left|D^{\prime}\right|+1$ gives $\gamma_{w n}(G) \leq \alpha_{1}(G)+\gamma_{R}(G)+1$.

Theorem 2.4: For any nontrivial tree $T, \gamma_{w n}(T) \leq i(T)+$ $\gamma_{\text {Res }}(T)+1$.

Proof: Suppose $D=\left\{v_{1}, v_{2}, \ldots \ldots \ldots, v_{n}\right\} \subseteq V(T)$ be the set of vertices such that $N[D]=V(T)$. Then $D$ is a minimal $\gamma-$ set of $T$.If $\langle D\rangle$ is a set of isolates, then $D$ itself is an independent dominating set of $T$. Otherwise, if there exists an edge $e$ in $D$ then $D-\{e\}$ forms an independent dominating set of $T$. Since $D$ is dominating set of $T$. If the set of vertices in $V-D$ is adjacent to at least one vertex of $D$ and at least one vertex of $V-D$, then $D$ itself is a $\gamma_{\text {Res }}-$ set of $T$.In lict graph $n(T)$, let $M=\left\{v_{1}, v_{2}, \ldots \ldots, v_{n}\right\} \subseteq V[n(T)]$ be the set of minimum degree vertices such that $N[M]=$ $V[n(T)]$.Then $M$ forms a minimal $\gamma_{w n}$-set of $T$. Hence $|M| \leq|D-\{e\}|+|D|, \quad$ gives $\gamma_{w n}(T) \leq i(T)+\gamma_{\text {Res }}(T)+1$.

\section{Lower Bounds for $\gamma_{w n}(G)$ :}

Here we have found lower bounds for $\gamma_{w n}(G)$ in terms of elements of $G$ and other domination parameters of $G$.

Theorem 2.5: For any connected $(p, q)$ graph G, $\gamma_{w n}(G) \geq \gamma(G)$.

Proof: Since the $V[n(G)]=E(G) \cup C(G), \mathrm{C}(\mathrm{G})$ a cutvertex set of $G$.Now we deal with two cases.

Case 1: Suppose $G$ is a nontrival tree. Then one can see that $\gamma_{w n}(G) \geq \gamma(G)$.

Case 2: Suppose $\mathrm{G}$ is not a tree. Then again it is true that $\gamma_{w n}(G) \geq \gamma(G)$.

Theorem 2.6: For any connected $(p, q) \operatorname{graph} G, \gamma_{w n}(G) \geq$ $\operatorname{diam}(G)-1$.

Proof: Let $D=\left\{e_{1}, e_{2}, e_{3}, \ldots, e_{m}\right\} \subseteq E(G)$ be the set of edges which constitute the diametral path in $G$. Then $|D|=$ $\operatorname{diam}(G)$. Farther consider $E=\left\{e_{1}, e_{2}, e_{3}, \ldots \ldots, e_{m}\right\}$; $C=\left\{c_{1}, c_{2}, \ldots \ldots, c_{n}\right\}$ be the set edges and cutvertices in $\mathrm{G}$. Then $V[n(G)]=E(G) \cup C(G)$. Let $S=\left\{u_{1}, u_{2}, u_{3}, \ldots, u_{n}\right\}$ be the minimum degree vertices in $n(G)$, which covers all the vertices in $n(G)$ such that $N[S]=V[n(G)]$. Thus $S$ forms a minimal weak dominating set of $n(G)$. It follows that $|S| \geq$ $|D|-1$. Hence $\gamma_{w n}(G) \geq \operatorname{diam}(G)-1$.

In [2],the author has defined the double domination. A dominating set $D \subseteq V(G)$ is said to be double dominating set of $G$, if every vertex of $V$ is dominated by at least two verities in $D$. The double domination number $\gamma_{d d}(G)$ of $G$ is the least cardinality of a double dominating set of $G$.

Theorem 2.7: For any $\operatorname{connected}(p, q)$ graph $\mathrm{G}$, then $\gamma_{w n}(G) \geq\left\lfloor\frac{\gamma_{d d}(G)}{\Delta(G)}\right\rfloor$.

Proof: Suppose $A=\left\{v_{1}, v_{2}, v_{3}, \ldots, v_{j}\right\} \subseteq V(G)$ be the set vertices with $\operatorname{deg}\left(v_{i}\right) \geq 2,1 \leq i \leq j$ such that $N[A]=V(G)$. Hence $A$ forms a minimal dominating set of $G$. $A$ set $A \subseteq$ $V(G)$ is a double dominating set, if for every vertex $v \in$ $V(G), A^{\prime}=|N[v] \cap A| \geq 2$.Now we consider the lict graph 
$n(G)$ of a graph $G$. Such that $S=\left\{v_{1}, v_{2}, v_{3}, \ldots, v_{k}\right\} \subseteq$ $V[n(G)]$ be the set of vertices with $\operatorname{deg} v_{l} \geq 2 ; 1 \leq l \leq k$ and if $N[S]=V[n(G)]$. Then $S$ forms a $\gamma-$ set of $n(G)$.Suppose there exists a set $S_{1} \subseteq V[n(G)]-S$ such that $\forall v_{i} \in S_{1}$, $\operatorname{deg}\left(v_{i}\right) \leq \operatorname{deg}\left(v_{j}\right), \quad \forall v_{j} \in S$. Then $S \cup S_{1}$ forms a minimal weak dominating set of $n(G)$. Suppose there exists at least one vertex $v \in A$ of maximum degree such that $\operatorname{deg}(v)=\Delta(G)$.Hence $\left|S \cup S_{1}\right| . \Delta(G) \geq|A|$. Clearly, it follows that $\gamma_{w n}(G) \geq\left\lfloor\frac{\gamma_{d d}(G)}{\Delta(G)}\right\rfloor$.

Theorem 2.8: For any tree $T$ with $K$ number of cut vertices then $\gamma_{w n}(T) \geq K$.

Proof: Let $B=\left\{c_{1}, c_{2}, c_{3}, \ldots, c_{n}\right\}$ be the set of all cutvertices in tree $T$, with $|B|=K$. Further let $J=\left\{e_{1}, e_{2}, e_{3}, \ldots, e_{i}\right\}$ be the set of all end edges in $T$, also $J^{\prime}=\left\{e_{1}^{\prime}, e_{2}^{\prime}, e_{3}^{\prime}, \ldots, e_{i}^{\prime}\right\}$ be the set of all nonend edges in $T$. Then in lict graph $n(T), V[n(T)]=J \cup J^{\prime} \cup B$. Suppose $D \subseteq V[n(T)]$ and $N[D]=V[n(T)] \quad$ and $\operatorname{deg}\left(v_{i}\right)<\operatorname{deg}\left(v_{j}\right) \quad$ where $\quad v_{i} \in$ $V[n(T)]-D$ and $v_{j} \in D$. Since $v_{i} \in D_{1}=V[n(T)]-D$, then $\left|D \cup D_{1}\right| \geq|K|$ which gives $\gamma_{w n}(T) \geq K$.

Theorem 2.9: For any non trivial tree $T$, vertices and $l$ end vertices and $S$ the number of support vertices, then $\gamma_{w n}(T) \geq$ $\left\lceil\frac{q-2-l+s}{3}\right\rceil$.

Proof: Let $I=\left\{u_{1}, u_{2}, u_{3}, \ldots, u_{m}\right\} \subseteq V(T)$ be the set of all end vertices in $T$ with $|I|=l$ and let $J=\left\{v_{1}, v_{2}, v_{3}, \ldots, v_{n}\right\}$ be the support vertices in $T$ with $|J|=S$. In lict graph $n(T), D=$ $\left\{v_{1}, v_{2}, \ldots, v_{i}\right\}=V[n(T)]$. Suppose $D_{1} \subseteq D, \quad \forall v_{j} \in D_{1}$, $\operatorname{deg}\left(v_{j}\right) \geq 2$ and $D_{2} \subseteq D$ be the set of minimum degree vertices which are adjacent to a cutvertex of $n(T)$, since each block of $n(T)$ is complete and covers all the vertices of $n(T)$. Then $\left\{D_{1} \cup D_{2}\right\}$ is a minimal weak dominating set of $n(T)$.Thus

$3\left|D_{1} \cup D_{2}\right| \geq|E(T)|-2-|I|+|J|$, gives $\gamma_{w n}(T) \geq$ $\left\lceil\frac{q-2-l+s}{3}\right\rceil$.

A dominating set $S \subseteq V(G)$ is a strong nonsplit dominating $s e t$, if the induced subgraph $\langle V-S\rangle$ is complete. The strong nonsplit domination number $\gamma_{s n s}(G)$ of $G$ is minimum cardinality of a strong nonsplit dominating set of $G$.

Theorem 2.10: For any connected $(p, q)$ graph $G$, with $p \geq 2$ then $\gamma_{w n}(G) \geq\left\lfloor\frac{\gamma_{\text {sns }}(G)+2}{3}\right\rfloor$ and $G \neq K_{1, n}(n \geq 4)$.

Proof: Suppose $G=K_{1, n}, n \geq 4, \gamma_{w n}(G)=1<\left|\frac{n+2}{3}\right|$, a contradiction to the fact.

Lat $D=\left\{v_{1}, v_{2}, \ldots \ldots \ldots, v_{n}\right\}$ be the vertex set of G. Suppose $D_{1}=\left\{v_{1}, v_{2}, \ldots \ldots \ldots, v_{m}\right\} \subseteq D$ and if $N\left[D_{1}\right]=V(G)$. Then $D_{1}$ is a minimal dominating set of $G$. If the induced subgraph $\left\langle V-D_{1}\right\rangle$ is complete, then $D_{1}$ itself is a $\gamma_{s n s}-$ set of $G$. Since the $V[n(G)]=E(G) \cup C(G), \mathrm{C}(\mathrm{G})$ a cutvertex set of $G$. Suppose $F=\left\{v_{1}, v_{2}, \ldots \ldots \ldots, v_{k}\right\} \subseteq V[n(G)]$ if $N[F]=V[n(G)] \quad$ and $\quad|\operatorname{deg}(x)-\operatorname{deg}(y)| \geq 2, \forall x \in$ $V[n(G)]-F, \forall y \in F$. Then $F$ forms a weak dominating set of $n(G)$. Otherewise there exists at least one vertex $\{w\} \subseteq$ $F$ such that $F \cup\{w\}$ forms a minimal $\gamma_{w n}-$ set. Thus $3|F| \geq\left|D_{1}\right|+2$. Hence $\gamma_{w n}(G) \geq\left\lfloor\frac{\gamma_{s n s}(G)+2}{3}\right\rfloor$.

Theorem 11: For any connected $(p, q)$ graph $G$, then $\gamma_{w n}(G)+\alpha_{0}(G) \geq \gamma(G)+\gamma_{s s}(G)$.
Proof: Let $M=\left\{v_{1}, v_{2}, \ldots \ldots \ldots, v_{n}\right\} \subseteq V(G)$ and $\forall e_{i} \in$ $E(G)$ is incident to at least one vertex of $M$. Then $|M|=\alpha_{0}$. Let $M_{1} \subseteq M$ Such that $N\left[M_{1}\right]=V(G)$ then $M_{1}$ is a minimal dominating set of G and $M_{2} \subseteq V(G)-M_{1}$. Suppose $M_{2}^{\prime} \subseteq M_{2}$ and $H=\left[V(G)-\left(M_{1} \cup M_{2}^{\prime}\right)\right]$ where $N\left[M_{1} \cup M_{2}^{\prime}\right]=V(G)$ and $\langle H\rangle$ is totally disconnected. Clearly $M_{1} \cup M_{2}^{\prime}=\gamma_{s s}-$ set of $G$. Now in $n(G)$, let $R=\left\{u_{1} u_{2}, \ldots \ldots \ldots, u_{n}\right\} \subseteq$ $V[n(G)]$ be the minimal set of vertices where $N[R]=$ $V[n(G)]$. Then $R$ is a dominating set of $n(G)$. Suppose $\forall v_{i} \in R_{1}=V[n(G)]-R, \operatorname{deg}\left(v_{i}\right) \leq \operatorname{deg}\left(v_{k}\right) \quad$ where $\forall v_{k} \in R$, then $\left\{R \cup R_{1}\right\}$ forms a minimal weak dominating set of $n(G)$.Clearly $\left|R \cup R_{1}\right|+|M| \geq\left|M_{1}\right|+\left|M_{1} \cup M_{2}^{\prime}\right|$, gives $\gamma_{w n}(G)+\alpha_{0}(G) \geq \gamma(G)+\gamma_{s S}(G)$.

Next, we give a bound of $\gamma_{w n}(G)$ of $\mathrm{G}$ with minimum edge degree $\delta^{\prime}$ and $\gamma_{w}(G)$.

Theorem 2.12: If $G$ is a $(p, q)$ connected graph then $\gamma_{w n}(G) \geq\left\lfloor\frac{\gamma_{w}(G)+\delta^{\prime}(G)}{3}\right\rfloor-1$ and $G \neq K_{1, n}(n>5)$

Proof: $\quad$ Suppose $\quad G=K_{1, n} n>5, \gamma_{w n}(G)=1<$ $\left\lfloor\frac{\gamma_{w}(G)+\delta^{\prime}(G)}{3}\right\rfloor-1$, a contradiction to the fact.

Let $S \subseteq V(G)$ where $\forall v_{i} \in S$ is adjacent to at least one vertex of $V(G)-S$. Then $S$ is a dominating set of $G$. Suppose there exists a set $H \subseteq V(G)-S$ such that $\forall v_{j} \in H$, deg $\left(v_{j}\right) \leq$ $\operatorname{deg}\left(v_{k}\right), \forall v_{k} \in S$. Then $S \cup H$ forms a minimal $\gamma_{w}-$ set of $G$.In $n(G), V[n(G)]=[E(G) \cup C(G)] . \quad$ Let $D=\left\{u_{1}, u_{2}, \ldots \ldots \ldots, u_{n}\right\} \subseteq V[n(G)]$ be the set of vertices in $n(G)$.Suppose $D^{\prime} \subseteq D$ be the set of vertices with $\operatorname{deg}(w) \geq$ $3, \forall w \in D^{\prime}$ such that $N\left[D^{\prime}\right]=V[n(G)]$. If $\forall v_{i} \in V[n(G)]-D^{\prime}$ with $\operatorname{deg}\left(v_{i}\right)<$ 3. Then $\left\{D^{\prime}\right\} \cup\left\{v_{i}\right\}$ forms a $\gamma_{w n}-$ set of $G$. Sience for any graph $G$ there exists at least one edge $e$ with $\mid$ dege $\mid=\delta^{\prime}(G)$. Hence $3\left|D^{\prime} \cup\left\{v_{i}\right\}\right|+1 \geq|S \cup H|+\mid$ dege $\mid$. Therefore $\gamma_{w n}(G) \geq\left\lfloor\frac{\gamma_{w n}(G)+\delta^{\prime}(G)}{3}\right\rfloor-1$.

The minimum number of color in any coloring of a graph $G$ such that no two adjacent vertices have same color is called the chromatic number of $G$ and is denoted by $\chi(G)$.

Theorem 2.13: If $\mathrm{T}$ is a tree, with $p \geq 2$, then $\gamma_{w n}(T) \geq$ $\gamma_{s}(T)+\chi(T)-2$.

Proof: Let $B_{1}=\left\{v_{1}, v_{2}, \ldots \ldots \ldots, v_{k}\right\} \subseteq V(T)$ be the set of all nonend vertices in $T$. Such that $N\left[B_{1}\right]=V(T)$ then $B_{1}$ forms a $\gamma-$ set of $T$. Suppose the induced subgraph $\left\langle V-B_{1}\right\rangle$ is have more than one component then $B_{1}$ itself is a split dominating set of $T$. In $n(T)$, let $A=\left\{v_{1}, v_{2}, \ldots \ldots \ldots, v_{m}\right\} \subseteq$ $V[n(T)]$ be the $\gamma_{w n}-$ set. For any tree, $\chi(T)=2$ and $\gamma_{w n}(T) \geq \gamma_{s}(T)+2-2$. Therefore $|A| \geq\left|B_{1}\right|+\chi(T)-2$, gives $\gamma_{w n}(T) \geq \gamma_{s}(T)+\chi(T)-2$.

A set $F \subset E(G)$ is called an edge dominating set of $G$, if every edge not in $F$ is adjacent to at least one edge in $F$. The minimum cardinality taken over all the edge dominating set of $G$, denoted as $\gamma^{\prime}(G)$ is called an edge domination number of $G$.

Theorem 2.14: For any connected $(p, q)$ graph $G$, with $p \geq$ 2 , then $\gamma_{w n}(G)+\Delta^{\prime}(G) \geq \gamma^{\prime}(G)+\left\lfloor\frac{\beta_{0}}{2}\right\rfloor-1$.

Proof: Let $E=\left\{e_{1}, e_{2}, \ldots \ldots \ldots, e_{k}\right\}$ be the edge set of $G$. Then $E_{n} \subseteq E$ such that $N\left[E_{n}\right]=E(G)$, then $E_{n}$ is an edge dominating set of $G$. Suppose $e \in E_{n}$ is an edge have maximum degree in $G$ such that $\operatorname{deg}(e)=\Delta^{\prime}(G)$. Let $A=$ 
$\left\{v_{1}, v_{2}, \ldots \ldots \ldots, v_{n}\right\} \subseteq V(G)$ be a maximal independent vertex set of $G$ such that $N\left(v_{i}\right) \cap N\left(v_{j}\right)=u, 1 \leq i \leq n$ and $1 \leq j \leq n$. Also $u \in V(G)-A$. Hence $|A|=\beta_{0}(G$.).In lict graph $n(G)$, let $L=\left\{v_{1}, v_{2}, \ldots \ldots \ldots, v_{n}\right\} \subseteq V[n(G)]$ such that $N[L]=V[n(G)]$ and if $\forall v_{l} \in L$ has degree at least 2 and $v_{k} \in V[n(G)]-L$ and $\operatorname{deg}\left(v_{k}\right) \leq \operatorname{deg}\left(v_{l}\right)$. Then $L \cup\left\{v_{l}\right\}$ forms $\gamma_{w n}$-set. Hence $\left|L \cup\left\{v_{l}\right\}\right|+|\operatorname{deg}(e)| \geq\left|E_{n}\right|+$ $\left\lfloor\frac{A}{2}\right\rfloor-1$, gives $\gamma_{w n}(G)+\Delta^{\prime}(G) \geq \gamma^{\prime}(G)+\left\lfloor\frac{\beta_{0}}{2}\right\rfloor-1$.

Theorem 2.15: For any connected $(p, q)$ graph $G$,then $\gamma_{w n}(G) \geq\left\lfloor\frac{\gamma_{t}(G)+\gamma_{c}(G)}{2}\right\rfloor$ and $G \neq C_{p}(p \geq 5)$.

Proof: Let $H$ be a dominating set of $G$.If the induced subgraph $\langle H\rangle$ has exactly one component then $H$ itself is a connected dominating set of $G$.Otherwise, if $H$ has more than one component then attach the minimum number of vertices $\left\{v_{i}\right\} \in V(G)-H, \forall v_{i}, \operatorname{deg}\left(v_{i}\right) \geq 2$, so that $S=H \cup$ $\left\{v_{i}\right\}$ forms exactly one component. Clearly $S=\gamma_{c}-$ set of $G$. Let $V_{1}=V(G)-H$ and if $J \subseteq V_{1}$ be the minimum set of vertices which are adjacent to at least one vertex of $H$.Then $<H \cup J\rangle$ is a $\gamma_{t}-$ set of $G$. Suppose $R$ be the vertex set of lict graph $n(G)$. Let $R_{1} \subseteq R$ be the set of vertices with minimum degree and $N\left[R_{1}\right]=V[n(G)]$. Then $R_{1}$ is a minimal $\gamma_{w n}-$ set. Hence $2\left|R_{1}\right| \geq|H \cup J| \cup|S|$, resulting in $\gamma_{w n}(G) \geq\left\lfloor\frac{\gamma_{t}(G)+\gamma_{c}(G)}{2} \mid\right.$.

One can easily check for $C_{p} p \geq 5$, which gives a contradiction.

Theorem 2.16: Let $T$ be a tree which is not a star, then $\gamma_{w n}(T) \geq \frac{\gamma_{\cot }(T)}{\gamma_{R e s}(T)}+\delta$.

Proof: Suppose $T=K_{1, n}$. Then $\gamma_{w n}(T)=1<\left|\frac{\gamma_{c o t}(T)}{\gamma_{\text {Res }}(T)}+\delta\right|$. Hence $T \neq K_{1, n}$. Let $S$ be a minimal dominating set of $T$. Suppose the subgraph $\langle V-S\rangle$ has no isolate then $\mathrm{S}$ itself is a $\gamma_{c o t}-$ set of $T$. Otherwise there exist a set $R \subseteq V(T)-S$ with $\operatorname{deg}\left(v_{j}\right)=0, \forall v_{j} \in R$. Add $\left\{v_{j}\right\} \subseteq V(T)-S$ to $\{R\}$, so that $\quad S \cup R$ forms a $\gamma_{\text {cot }}-$ set.Suppose $V_{1}=\left\{v_{1}, v_{2}, \ldots \ldots \ldots, v_{n}\right\}$ be the set of end vertices in $T$.Now $\forall v_{i} \in V(T)-\left\{S \cup V_{1}\right\}$ is adjacent to at least one vertex of $S \cup V_{1}$ and at least one vertex of $V(T)-\left\{S \cup V_{1}\right\}$. Then $\left\{S \cup V_{1}\right\} \quad$ is a $\quad \gamma_{\text {Res }}-$ set of T. Suppose $D=\left\{v_{1}, v_{2}, \ldots \ldots \ldots, v_{m}\right\} \subseteq V[n(T)]$ and $\operatorname{deg}\left(v_{m}\right) \leq \operatorname{deg}\left(v_{k}\right)$, $\forall v_{k} \in V[n(T)]-D$ and $\forall v_{m} \in D$ such that $N\left[v_{m}\right]=$ $V[n(T)]$. Then $D$ forms a $\gamma_{w n}-$ set of $T$. Clearly $|D| . \mid S \cup$ $V_{1}|-\delta \geq| S \cup R \mid$ resulting into $\gamma_{w n}(T) \geq \frac{\gamma_{c o t}(T)}{\gamma_{R e s}(T)}+\delta$.

\section{REFERENCES}

[1] F. Harary, Graph Theory, Addison-Wesley, Reading Mass, 1974

[2] F. Harary and T. W. Haynes, Double domination in graphs, Ars combin, Vol-55, 2000, 201- 213

[3] T. W. Haynes, S. T. Hedetniemi and P. J. Slater, Fundamentals of Domination in Graph, Marcell Dekker, INC-1998.

[4] T. W. Haynes, S. T. Hedetniemi, P. J. Slater (Eds), Domination in graph: Advanced Topics, Marcel Dekker, INC, New York, 1998

[5] V.R. Kulli, Theory of domination in Graphs, Viswa international publications, 2010

[6] V R. Kulli and M. H. Muddebihal Lict and Litact graph of a graphs, journal of analysis and computation, Vol-2, 2006, 133-143.

[7] M. H. Muddebihal and Geetadevi Baburao, weak line domination in graph theory, IJRAR Vol-6, 2019, 129133.

[8] E. Sampathkumar and Puspa Latha, strong weak domination number and domination balance in graph, Discrete Math, Vol-161, 1996, 235-242. 